\title{
Ventricular Enlargement in Schizophrenia Related to Volume Reduction of the Thalamus, Striatum, and Superior Temporal Cortex
}

\author{
Christian Gaser, M.E.E., Ph.D. \\ Igor Nenadic \\ Bradley R. Buchsbaum, Ph.D. \\ Erin A. Hazlett, Ph.D. \\ Monte S. Buchsbaum, M.D.
}

Objective: Enlargement of the lateral ventricles is among the most frequently reported macroscopic brain structural changes in schizophrenia, although variable in extent and localization. The authors investigated whether ventricular enlargement is related to regionally specific volume loss.
Method: High-resolution magnetic resonance imaging scans from 39 patients with schizophrenia were analyzed with deformation-based morphometry, a voxel-wise whole brain morphometric technique.

Results: Significant negative correlations with the ventriclebrain ratio were found for voxels in the left and right thalamus and posterior putamen and in the left superior temporal gyrus and insula.

Conclusions: Thalamic shrinkage, especially of medial nuclei and the adjacent striatum and insular cortex, appear to be important contributors to ventricular enlargement in schizophrenia.

(Am J Psychiatry 2004; 161:154-156)

$\mathrm{E}$ the earliest reported structural brain imaging abnormalities found in schizophrenia, as well as one of the most stable findings in morphometric investigations (1). However, it is unclear whether ventricular changes are related to focal or diffuse volume reduction of brain parenchyma. Previous studies have implicated preferential enlargement of certain parts of the ventricular system, such as the temporal horn or body of the ventricles (2). This might suggest that ventricular enlargement in these patients is related to specific shrinkage of gray matter/white matter structures, rather than diffuse brain atrophy. Structures implicated in schizophrenia such as the thalamus, hippocampus, or corpus callosum are located adjacent to or near the ventricles and show structural alteration in schizophrenia (1). Here we tested the hypothesis that ventricular enlargement in schizophrenia is related to focal volume loss. Deformation-based morphometry $(3,4)$ was applied to obtain statistical parametric maps indicating areas of volume change correlated to ventricle-brain ratio (VBR) as a measure of ventricular enlargement.

\section{Method}

High-resolution $\mathrm{T}_{1}$-weighted magnetic resonance imaging scans of 39 patients (12 women, 27 men) who met DSM-III-R criteria for schizophrenia were obtained with a GE Signa $5 \mathrm{x}$ system (General Electric Medical Systems, Milwaukee) using a spoiled gradient recall acquisition pulse sequence ( $\mathrm{TR}=24 \mathrm{msec}, \mathrm{TE}=5$ msec, flip angle $=40^{\circ}$ ) with contiguous $1.2-\mathrm{mm}$ thick axial slices (in-plane matrix: $256 \times 256$, field of view $=23 \mathrm{~cm}$, voxel dimension: $0.89 \times 0.89 \times 1.2 \mathrm{~mm})$. The patients, a group studied in a previous validation report on deformation-based morphometry (4), ranged in age from 18 to 65 years (mean=37.2 years, $\mathrm{SD}=12.14$ ); 34 were right-handed, two were left-handed, and three were ambi- dextrous. Thirty-seven of the patients were evaluated with the 18item Brief Psychiatric Rating Scale (BPRS) (mean score $=50.3$ [SD= 12.7 , range $=30-83$, minimum possible rating $=18]$ ). Diagnosis of schizophrenia $(\mathrm{N}=35)$ or schizoaffective disorder $(\mathrm{N}=4)$ was determined with a structured psychiatric interview, the Comprehensive Assessment of Symptoms and History (5). Individuals with a history of substance abuse/dependence, neurological disorder, or head trauma were excluded. All patients were assessed for their capacity to give consent by a board-certified psychiatrist, and written informed consent was obtained for a protocol approved by the local institutional review board.

We first obtained volume measures of the lateral ventricles by using a semimanual tracing procedure implemented in the MultiImage Processing Software package (Neuroscience PET Laboratory, Mount Sinai School of Medicine, New York) and calculated VBRs as a normalized measure (2). The reliability of this procedure was assessed by outlining ventricles in eight subjects by two tracers (intraclass correlation $=0.98$ [2]).

For deformation-based morphometry analysis, we first applied a linear, affine normalization algorithm (SPM 99 software, Institute of Neurology, London) for reorientation of images in space and correction of overall brain width, height, and length without changing local anatomy of the brains, thus transforming images to a standard stereotactic space equivalent to Talairach space (6). Then we applied nonlinear normalization, warping every subject brain to a single template brain through a multigrid algorithm (7). From resulting three-dimensional deformation fields (consisting of three-dimensional displacement vectors in every voxel of the image), we computed the Jacobian determinant to obtain voxelwise maps of local volume change (4) relative to the template. For statistical analysis, we applied a general linear model (multiple regression with VBR as a covariate, equalling a voxel-wise correlation analysis) to examine pixel by pixel the relationship between VBR and local volume throughout the brain. To remove variance related to age effects we entered age into the model as a confounding variable.

For resulting statistics, we set the significance threshold for height at $\mathrm{p}<0.05$ (corrected for multiple comparisons, according to the false discovery rate approach [8]), which corresponded to 
FIGURE 1. Regions of Positive and Negative Correlations Between Ventricle-Brain Ratio and Deformation-Based Morphometry-Derived Volume in 39 Patients With Schizophrenia ${ }^{a}$

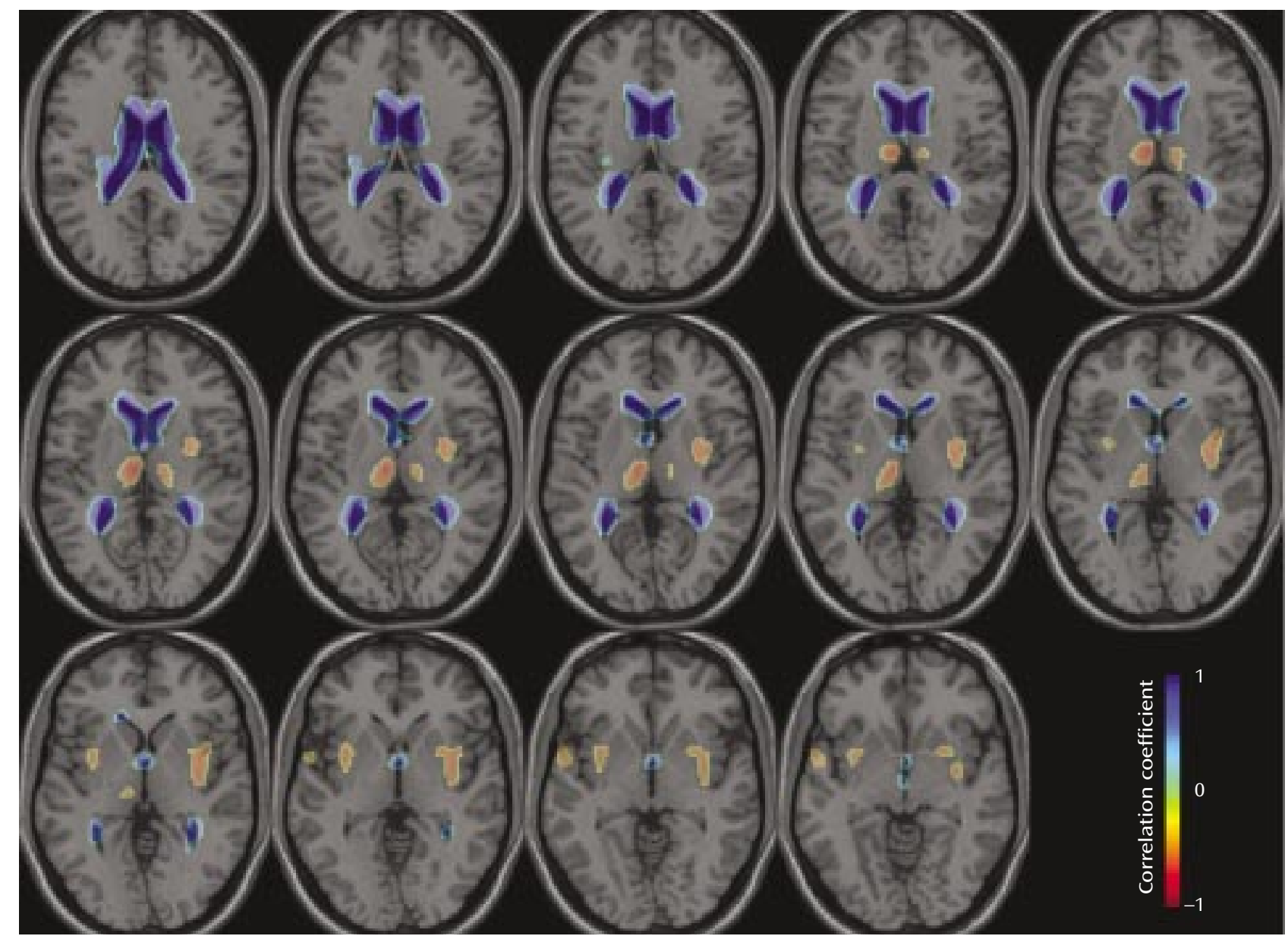

a Images are axial slices from -6 to $20 \mathrm{~mm}$ according to Talairach coordinates. A negative correlation means that locale volume decreases with larger values of VBR. Correlational significance ( $p<0.05$, corrected for multiple comparisons) corresponded to $r>0.55$ and $r<-0.55$; spatial extent significance $(p<0.05)$ corresponded to 39 voxels.

values of $\mathrm{r}<-0.55$ and $\mathrm{r}>0.55$. We set significance at $\mathrm{p}<0.05$ for extent, which corresponded to 39 voxels.

\section{Results}

Positive correlations of VBR and local Jacobian determinant were found for the lateral ventricles (maximum voxel: $\mathrm{x}=-12, \mathrm{y}=-10, \mathrm{z}=26 ; \mathrm{r}=-0.93, \mathrm{df}=36, \mathrm{p}<0.001$ ) as expected (4) but also parts of the third ventricle. A positive correlation means that local volume increases with larger VBR values. For negative correlations (higher VBR related to local shrinkage) (Figure 1), the strongest effects were found in the left medial/posterior thalamus $(\mathrm{x}=-8, \mathrm{y}=-16, \mathrm{z}=12$; $\mathrm{r}=-0.80, \mathrm{df}=36, \mathrm{p}<0.001)$, right thalamus $(\mathrm{x}=12, \mathrm{y}=-16, \mathrm{z}=$ $12 ; \mathrm{r}=-0.66, \mathrm{df}=36, \mathrm{p}<0.01$ ), left superior temporal gyrus $(\mathrm{x}=-58, \mathrm{y}=2, \mathrm{z}=-4 ; \mathrm{r}=-0.61, \mathrm{df}=36, \mathrm{p}<0.01)$, left putamen $(\mathrm{x}=$ $-26, y=6, z=-12 ; r=-0.72, d f=36, p<0.01)$ and the right putamen $(x=36, y=-10, z=0 ; r=-0.67, d f=36, p<0.01)$. The shrinkage in the putamen may extend into the insula. Some small clusters (right middle temporal gyrus, posterior cingulate, right cerebellum) comprised only few voxels, thus failing to reach the spatial extent threshold of $\mathrm{p}<0.05$.
No significant differences were found in hippocampal areas adjacent to the temporal horns of the ventricles or the corpus callosum.

\section{Discussion}

This study demonstrates that larger ventricles within a population of schizophrenia patients are associated with regionally specific reductions of brain parenchyma in both paraventricular and remote areas. Strongest effects were found in the thalamus, which is adjacent to the body of the lateral ventricles and might have a principal role in schizophrenia. It is interesting that the significant voxels were located in parts of the internal lamina, the mediodorsal nuclei, and the pulvinar nuclei of the thalamus. This coincides with the previous finding of the mediodorsal and pulvinar nuclei, two major relay nuclei to prefrontal and temporal cortices, being reduced in volume in schizophrenia (9).

The putamen and superior temporal gyrus findings demonstrate that remote brain areas also might be involved or associated with ventricular enlargement in 
schizophrenia. Changes in the putamen might, however, also be induced by neuroleptic medication (1), which increases rather than decreases size. Several studies have not shown ventricular size to be correlated with drug treatment history (10), unlike striatal size or thalamic size (11). Thus, it appears that medication effects might tend to obscure or diminish ventricle-thalamus size correlations.

These results contradict the notion that large ventricles in schizophrenia simply reflect diffuse brain atrophy (12). Rather, focal shrinkage in distinct gray matter regions involved in the pathophysiology of the disorder and adjacent to the ventricles might be a main contributor to this effect. This could also explain the variability in localization and extent of ventricular changes in schizophrenia. While it is possible that a population of normal comparison subjects might show similar thalamic ventricular volume linkage, the limited incidence of ventricular enlargement in normal subjects in this age range make this finding less probable. However, the recent data of Chance et al. (13) revealed negative correlations between temporal lobe volume and ventricular volume (similar to ours) and low and positive correlations within their normal group.

Received Dec. 27, 2002; revision received June 17, 2003; accepted July 21, 2003. From the Department of Psychiatry, Friedrich-SchillerUniversity of Jena; the Neuroscience PET Laboratory, Department of Psychiatry, Mount Sinai School of Medicine, New York; and the NIMH Clinical Brain Disorders Branch, Bethesda, Md. Address reprint requests to Dr. Gaser, Department of Psychiatry, Friedrich-SchillerUniversity of Jena, Philosophenweg 3, D-07743 Jena, Germany; Christian.Gaser@uni-jena.de (e-mail).

This work was supported by NIMH grants $\mathrm{MH}-40071, \mathrm{MH}-60023$, and $\mathrm{MH}-56489$ (M.S.B.) and grants from the Dana Foundation (M.S.B.), the National Alliance for Research on Schizophrenia and Depression (E.A.H.), and the Friedrich-Schiller-University of Jena (VKF Clinical Research Council grant [C.G.] and AAA travel grant [I.N.]).

\section{References}

1. Wright IC, Rabe-Hesketh S, Woodruff PWR, David AS, Murray RM, Bullmore ET: Meta-analysis of regional brain volumes in schizophrenia. Am J Psychiatry 2000; 157:16-25
2. Buchsbaum MS, Yang S, Hazlett E, Siegel BV Jr, Germans M, Haznedar M, O'Flaithbheartaigh S, Wei T, Silverman J, Siever LJ: Ventricular volume and asymmetry in schizotypal personality disorder and schizophrenia assessed with magnetic resonance imaging. Schizophr Res 1997; 27:45-53

3. Gaser C, Volz HP, Kiebel S, Riehemann S, Sauer H: Detecting structural changes in whole brain based on nonlinear deformations-application to schizophrenia research. Neuroimage 1999; 10:107-113

4. Gaser C, Nenadic I, Buchsbaum BR, Hazlett EA, Buchsbaum MS: Deformation-based morphometry and its relation to conventional volumetry of brain lateral ventricles in brain MRI. Neuroimage $2001 ; 13: 1140-1145$

5. Andreasen NC, Flaum M, Arndt S: The Comprehensive Assessment of Symptoms and History (CASH): an instrument for assessing diagnosis and psychopathology. Arch Gen Psychiatry 1992; 49:615-623

6. Talairach J, Tournoux P: Co-Planar Stereotaxic Atlas of the Human Brain: Three-Dimensional Proportional System. New York, Thieme Medical, 1988

7. Kjems U, Strother SC, Anderson J, Law I, Hansen LK: Enhancing the multivariate signal of $\left[{ }^{15} \mathrm{O}\right]$ water PET studies with a new nonlinear neuroanatomical registration algorithm. IEEE Trans Med Imaging 1999; 18:306-319

8. Benjamini Y, Hochberg Y: Controlling the false discovery rate: a practical and powerful approach to multiple testing. J R Stat Soc B 1995; 57:289-300

9. Byne W, Buchsbaum MS, Kemether E, Hazlett EA, Shinwari A, Mitropoulou V, Siever LJ: Magnetic resonance imaging of the thalamic mediodorsal nucleus and pulvinar in schizophrenia and schizotypal personality disorder. Arch Gen Psychiatry 2001; 58:133-140

10. Gur RE, Turetsky BI, Bilker WB, Gur RC: Reduced gray matter volume in schizophrenia. Arch Gen Psychiatry 1999; 56:905911

11. Gur RE, Maany V, Mozley PD, Swanson C, Bilker W, Gur RC: Subcortical MRI volumes in neuroleptic-naive and treated patients with schizophrenia. Am J Psychiatry 1998; 155:1711-1717

12. Symonds LL, Archibald SL, Grant I, Zisook S, Jernigan TL: Does an increase in sulcal or ventricular fluid predict where brain tissue is lost? J Neuroimaging 1999; 9:201-209

13. Chance SA, Esiri MM, Crow TJ: Ventricular enlargement in schizophrenia: a primary change in the temporal lobe? Schizophr Res 2003; 62:123-131 